\title{
La aplicación de Zeolita en la producción avícola: Revisión
}

\author{
Hernán Collazos García \\ hernan.collazos@unad.edu.co \\ Universidad Nacional Abierta y a Distancia UNAD \\ Escuela de Ciencias Agrícolas, Pecuarias y del Medio Ambiente \\ CEAD José Celestino Mutis, Bogotá D.C. Colombia
}

\begin{abstract}
Resumen.- El objetivo de la presente revisión es estudiar y discutir las aplicaciones de las Zeolitas en la producción avícola. Las Zeolitas son aluminosilicatos cristalinos que presentan una estructura tridimensional infinita, a las cuales se les atribuyen numerosas propiedades como su capacidad para perder y ganar agua reversiblemente y ser capaces de intercambiar selectivamente una variedad de cationes sin presentar cambios mayores en su estructura básica; esto las hace potencialmente útiles en la nutrición animal. Sin embargo el efecto de las Zeolitas en el consumo de alimento, ganancia de peso, tasa de crecimiento, producción de huevos, peso del huevo, grosor de la cáscara y características internas del huevo están aún en discusión. Mientras que algunos de los experimentos reportan efecto benéfico luego de su inclusión en las dietas para aves, otros muestran que no hay variaciones o que pueden producir efectos negativos. Las variaciones en la proporción de uso, el tipo de Zeolita (natural o sintética) y el contenido de impurezas, pueden ser los responsables de las discrepancias reportadas en los experimentos.
\end{abstract}

Palabras clave: Aflatoxinas, Avicultura, Dieta, Producción, Zeolita.

\begin{abstract}
The aim of this review is to study and discuss the use of Zeolite in poultry production. Zeolites are crystalline, hydrated aluminosilicates of alkali and alkaline cations which have infinite, three-dimensional structure. Zeolites can lose and gain water reversibly and they are capable of selectively exchange a variety of cations with no major structural changes. These characteristics make them suitable for animal nutrition. However the effect of Zeolites on food intake amounts, weight gain, growth rate, egg production, egg weight, shell thickness and internal egg quality are still under discussion. While some sort of experiments report beneficial effects due to inclusion of Zeolite in the diet of birds, other experiments show no changes and yet there are results that indicate some negative effects. Reasons such as level of usage, type of Zeolite (natural or synthetics) and the levels of impurities in natural Zeolites, are thought to be responsible for reported discrepancies.
\end{abstract}

Key words: Aflatoxicosis, performance, Poultry, Zeolite.

\section{Introducción}

Los primeros reportes de los efectos de las Zeolitas naturales sobre la producción animal fueron presentados por Mumpton y Fishman en la década de 1970 (Mumpton \& Fishman 1977). Evans (1989) trató con gran profundidad y detalle los efectos de las Zeolitas en la producción y desempeño avícola y en la década de 1990 había más de 60 artículos que trataban específicamente sobre aplicación de
Zeolitas en la producción avícola y hubo mucho entusiasmo por este nuevo ingrediente alimenticio, a pesar del descubrimiento de su papel en la aflatoxicosis. (Ramous \& Gounzales 1997), que hizo pensar que estas no eran tan promisorias como parecían.

La intención de esta revisión no es discutir la química y estructura de la Zeolita, como lo hicieron Mumpton \& Fishman (1977), ni discutir en detalle los experimentos reporta- 
dos por Ramous \& Gounzales (1997). Esta revisión pretende reunir información actualizada sobre las Zeolitas y su relación con la avicultura, además de discutir aquellas propiedades que pueden ser explotadas en esta cadena de producción. La información presentada es la más relevante y tiene la suficiente profundidad para los investigadores y miembros de la comunidad académica que se interesen en este tema.

Definición y Estructura.- Las Zeolitas fueron descubiertas por primera vez en 1756 por el Barón Axel Fredrick Constedt, quien ta nombró a partir de las palabras griegas zein (hervir) y lithos (piedra), es decir "piedra hirviente", con lo cual se describe la presencia de moléculas de agua en las cavidades de Zeolitas naturales y alude a su peculiar característica de burbujear cuando se calientan.

Las Zeolitas son aluminosilicatos cristalinos, hidratados de álcalis $\left(\mathrm{Na}^{+}, \mathrm{K}^{+}, \mathrm{Rb}^{+}, \mathrm{Ca}^{+}\right)$y cationes alcalino-térreos $\left(\mathrm{Be}^{+2}, \mathrm{Mg}^{+2}, \mathrm{Ca}^{+2}, \mathrm{Sr}^{+2}\right)$ con una estructura tridimensional potencialmente infinita. Gottardi \& Galli (1985) las presentaron con la fórmula general:

$$
M_{2 / n} \mathrm{O}_{2} \mathrm{Al}_{2} \mathrm{O}_{3} x \mathrm{SiO}_{2} \cdot y \mathrm{H}_{2} \mathrm{O} \text {. }
$$

Donde $M$ es cualquier álcali o catión alcalino-terreo, $n$ es la valencia del catión, $x$ es un número de 2 a 10 y $y$ un número de 2 a 7 . Cada especie de Zeolita tiene una estructura propia única que puede ser visualizada como un tetraedro de $\mathrm{SiO}_{4}$ y $\mathrm{AlO}_{4}$, ligados en una forma geométrica simple.

Propiedades y funciones.- Las propiedades de las Zeolitas se han venido describiendo desde comienzo de los años 70's e incluyen la capacidad para ganar y perder agua reversiblemente, una baja densidad y una estructura cristalina generalmente estable al ser deshidratadas. También presentan un intercambio catiónico que no implica cambios básicos en su estructura, adsorción de gases o vapor, así como catálisis y canales moleculares uniformes en cristales deshidratados.

Cada especie de Zeolita tiene unas características químicas y físicas propias, que incluyen la estructura cristalina, tamaño, forma de cavidades, porosidad y dureza de la roca madre (Pond \& Lee 1984). Esta diversidad de propiedades ha hecho que las Zeolitas se empleen en muchos sectores industriales, pues sus usos incluyen el control de olores, el tratamiento de aguas, el control de la polución, la agricultura, la horticultura y la alimentación animal.

En cuanto a la nutrición animal, existen dos características que determinan su efectividad. En primer lugar está su capacidad de adsorción, pues las Zeolitas tienen la capacidad de perder y ganar agua reversiblemente. En segundo lugar está el intercambio iónico, en el cual se hace un intercambio de cationes sin cambios básicos en su estructura.

\section{Procesamiento y síntesis de Zeolitas.-} Desde su descubrimiento hace más de 200 años, se han reconocido alrededor de 85 Zeolitas naturales, a las que se suman al menos 100 especies sintetizadas en el laboratorio, de las que no se conoce una contraparte natural. Las Zeolitas naturales y sintéticas son diferentes en sus características físicas y químicas. Una diferencia importante es la relación de sílice-aluminio, la cual es aproximadamente de 2.5-5: 1 para Zeolitas naturales y 1:1 para sintéticas. La Zeolita sintética más comúnmente utilizada, la Zeolita $\mathrm{A}$, tiene la composición $\left.\mathrm{Na}_{12}\left[\mathrm{AlO}_{2}\right) 12\left(\mathrm{SiO}_{2}\right)\right] .24 \mathrm{H}_{2} \mathrm{O}$ y la natural más común, conocida como Clinoptilolita, tiene la composición $\mathrm{CaNa}_{4} \mathrm{~K}_{4}\left(\mathrm{AlO}_{2}\right)_{5}\left(\mathrm{SiO}_{2}\right)_{30} .24 \mathrm{H}_{2} \mathrm{O}$.

Esta relación sílice-aluminio afecta la estabilidad de las Zeolitas bajo diferentes condiciones de $\mathrm{pH}$. Las Zeolitas naturales son más estables que las sintéticas y no se descomponen en un ambiente ligeramente ácido, manteniendo su estructura gracias al mayor contenido de sílice. Por su parte, el aluminio contenido en la molécula de una Zeolita sintética se vuelve soluble y amarra el fósforo dietético, lo que hace que el fosfato de aluminio sea insoluble en el tracto gastrointestinal, reduciendo la disponibilidad de fósforo (Elliot et al. 1990). Además el aluminio liberado puede causar envenenamiento. La adición de un $1 \%$ de Zeolita sintética significa inclusión de $0.15 \%$ de aluminio en la dieta, lo que constituye un nivel tóxico para pollos de engorde y gallinas ponedoras (Edwards et al. 1992).

Un trabajo posterior demostró que el aluminio se acumula en la tibia de pollitos alimentados con Zeolita sintética (Leach et al. 1990). Sin embargo no se encontraron diferencias en el contenido de aluminio en los pulmones, cerebro, riñones e hígado en aves con inclusión de 
Zeolita en la dieta y aquellas con tratamiento testigo, a pesar de la posibilidad de que las Zeolitas puedan ser descompuestas en el proventrículo del tracto digestivo y de que el aluminio pueda filtrarse.

Las Zeolitas sintéticas pueden contener también contaminantes desde su manufactura, como el hidróxido de sodio que es un álcali fuerte que puede causar variaciones en el $\mathrm{pH}$ del intestino de las aves. Se ha reportado que el sodio dentro de la molécula de la Zeolita sintética tiene el mismo nivel de disponibilidad para el pollo que el que presenta el cloruro de sodio (Fethiere et al. 1988).

\section{Efecto de las Zeolitas en la producción avícola}

Consumo de Alimento.- El efecto de la adición de Zeolitas en la dieta sobre las tasas de consumo de alimento varía. Oliver (1989) reportó un aumento en el consumo, mientras que Roland y colaboradores (1985) no encontraron ninguna variación y Miles y colaboradores (1986) reportaron que las gallinas ponedoras que consumieron Zeolitas requerían menos cantidad de alimento. Una de las razones de tal discrepancia en los resultados podría ser el balance de la dieta. Una proporción de hasta $10 \%$ de Zeolitas en la dieta (como puede hacerse cuando se usa Zeolita natural) produce cambios en la composición y concentración de algunos elementos en la dieta y de este modo cambios en el contenido de energía, proteínas y aminoácidos.

Las Zeolitas adicionadas pueden contener calcio, aluminio, sodio y otros elementos que pueden afectar el balance mineral y por lo tanto no es posible determinar si los cambios son producidos por la Zeolita como tal, o por estos elementos. El balance de la dieta para minerales y el balance anión-catión, así como la proteína y energía, son esenciales para asegurar que las verdaderas características de la Zeolita son evaluadas y no confundidas con efectos de dilución de dietas no balanceadas.

Ganancia de peso y tasa de crecimiento.- Se ha pretendido justificar el uso de Zeolitas en la dieta de aves con el argumento de que tiene un efecto benéfico en la ganancia de peso. Sin embargo los resultados en este aspecto también presentan ambigüedades y conflictos, sin que haya un consenso general acerca de si la
Zeolita puede mejorar el crecimiento de pollos de engorde. Evans (1989) revisó 39 artículos que reportaban datos de ganancia de peso $y$ tasas de crecimiento, en los que se concluye que excepto en algunos casos que mostraron mejoras ligeras en el crecimiento de pollos de engorde, en la mayoría no se registró ningún efecto benéfico e incluso hubo un par de experimentos en los que se registró un efecto adverso.

Producción de huevos.- Casi todos los experimentos que evaluaron el peso del huevo, producción, masa, cáscara y calidad interna, reportaron una ausencia de beneficios luego de la inclusión de Zeolita en la dieta. De hecho la mayoría de experimentos mostraron un efecto negativo en dietas con un contenido mineral adecuado (Elliot \& Edwards 1991), mientras que Roland (1988) reportó que con un 1.5\% de Zeolita sintética aumentó la producción de huevos cuando el nivel de fósforo estaba por debajo de los requerimientos. Según Leach y colaboradores (1990) las Zeolitas son más efectivas en dietas bajas en calcio y parece que pueden tener efectos benéficos en dietas con deficiencias de otros contenidos minerales, por lo que puede pensarse que la utilización de una proporción adecuada de Zeolita puede reducir la utilización de dichos minerales en la dieta de las aves.

Desempeño óseo.- Leach y colaboradores (1990) reportaron que la Zeolita tenía efectos benéficos en el contenido de cenizas y la resistencia ósea, mientras que Elliot y colaboradores (1990) reportaron que, aunque la Zeolita aumentó los niveles de ceniza en piernas, no hubo efectos sobre la discondroplasia tibial. El más claro efecto de la Zeolita sobre la osteomalacia se mostró al suministrar una dieta baja en calcio y fósforo; aunque en dietas con niveles bajos de este elemento la inclusión de Zeolita produjo un aumento del contenido de aluminio en los huesos.

La Zeolita puede reducir los efectos adversos de los niveles altos de calcio en la dieta. Esto puede sugerir que la Zeolita ejerce su efecto en el pollo por intercambio iónico reversible de calcio, manteniéndolo en la forma disponible y por lo tanto mejorando la utilización mineral. Sin embargo, Leach y Edwards (1991) sugirieron que las Zeolitas pueden reducir la disponibilidad de fósforo en vez de aumentar la utilización del calcio en la dieta. 
Eficiencia alimenticia y utilización de nutrientes.- El mejor atributo dado a la Zeolita es el efecto benéfico sobre la eficiencia alimenticia tanto en pollos como en ponedoras. Parece haber un consenso general en este aspecto (Oliver 1997), aunque unos pocos reportes sugieren que la Zeolita no tuvo efectos benéficos (Vetz \& Shultz 1984) o que incluso tuvo un efecto negativo sobre este parámetro (Nakaue \& Koellikker 1981).

Los efectos de las Zeolitas sobre la eficiencia alimenticia podrían ser debidos a una reducción en la velocidad de paso en el intestino, la inmovilización de enzimas y su influencia en la microflora del intestino (Petunkin 1991). La posible mejora en la utilización de nutrientes puede ser atribuida a una reducción o predistribución de la microflora del intestino. Oliver (1989) reportó que el conteo bacterial en las secciones distal y proximal del intestino fue significativamente más bajo cuando se había proporcionado una dieta con Zeolita.

Las Zeolitas pueden facilitar el drenaje de sangre de las vellosidades y aumentar la actividad de las células que las bordean, las cuales a su vez podrían mejorar la digestión y absorción de nutrientes (Nestorov 1984, Petunkin 1991). Además las Zeolitas mejoran la energía metabolizable aparente y neta y la digestión verdadera de la proteína en el tracto digestivo (buche, proventrículo, molleja, intestino delgado y grueso), así como el número, tamaño y forma de las vellosidades intestinales; además pueden traer beneficios en los órganos del metabolismo como hígado, páncreas y riñones.

Consumo de agua.- Entre los diversos estudios que consideran los efectos de las Zeolitas en todos los aspectos del desempeño de las aves, ha habido pocos intentos de evaluar si existe algún efecto en el consumo de agua. Onagi (1966 en Mumpton \& Fishman 1977) demostró que el consumo de agua y el contenido de humedad de la cama se redujeron cuando se incluyó Zeolita en la dieta de pollos de engorde; sin embargo no hubo un análisis sobre los posibles efectos adversos del consumo de Zeolita sobre el consumo de agua.

Efecto sobre la cama y la condición de las excretas.- Una de las primeras funciones y propiedades atribuidas a la Zeolita es su capacidad de retener agua, por lo que uno de sus primeros usos fue para mejorar las condiciones de la cama. En 1966 los granjeros japoneses regaban Zeolita y estiércol en sus granjas, o la colocaban en el cielorraso, para combatir tanto la humedad como el mal olor en sus galpones. La base científica de tales aplicaciones fue primero probada y confirmada por Hayburst y Willard (Evans1989). Oliver (1997) reportó que el consumo de Zeolita origina un menor contenido de humedad en las heces y de este modo también en la cama. Las Zeolitas también fueron utilizadas para reducir las pérdidas de nitrógeno, en forma de amoniaco $\left(\mathrm{NH}_{3}\right)$, en el compostaje del estiércol de aves (Kithome et al. 1999).

Uso a alta temperatura ambiental y estrés calórico.- Aparentemente la Zeolita puede reducir la velocidad de respiración (i.e. el jadeo) que se produce por las altas temperaturas (Roussel et al. 1992). Según Ingram y colaboradores (1988) las aves sometidas a estrés calórico se beneficiaron con la inclusión de Zeolitas sintéticas en sus dietas, aunque Evans y colaboradores (com. pers.) no encontraron ningún efecto benéfico en aves sometidas a tales condiciones de estrés.

Aflatoxinas.- Las aflatoxinas son un grupo de químicos tóxicos producidos por ciertas especies de hongos del género Aspergillus, que comúnmente se presentan como contaminantes naturales de los alimentos para aves. La toxicidad en el alimento de las aves ha sido bien documentada (Huff et al. 1988) y se ha reportado una gran variedad de métodos físicos, químicos, biológicos y nutricionales para tratar la aflatoxicosis, con diferentes niveles de éxito. La inclusión de $0.5 \%$ de aluminosilicato hidratado de calcio y sodio (HSCAS) de una Zeolita natural en la dieta logró una protección casi total contra la aflatoxina B1 en pollos y ponedoras (Kubena et al. 1993). Se sabe además que las Zeolitas reducen la absorción de micotoxinas en el tracto gastrointestinal, evitando los efectos tóxicos observados en el ganado y el paso de las toxinas a los productos animales que entran a la cadena alimenticia (Kubena \& Harvey 1991). Sin embargo la efectividad del uso de HSCAS como preventivo para otros tipos de aflatoxinas es menor (Ramous \& Gounzales 1997).

Zeolitas vs. Toxicidad.- La capacidad de intercambio catiónico de las Zeolitas puede reducir la toxicidad originada por ingestión de metales pesados (Bartko et al.1995, Pond 
et al. 1995). Sin embargo la misma Zeolita constituye una fuente de toxicidad, dada la probabilidad de que al descomponerse en el proventrículo o molleja queden residuos de aluminio disuelto; no obstante, esto no es una constante y no ha ocurrido en todos los experimentos realizados. Por otra parte, el alto nivel de aluminio presente en la Zeolita natural puede resultar tóxico, Elliot \& Edwards (1991) indicaron que el aluminio contenido en las moléculas de Zeolita sintética pueden ser solubles en el tracto gastrointestinal de ponedoras y pollos de engorde.

Raza, edad y sexo.- No se ha probado la relación entre el sexo y edad de las aves con las respuestas productivas a las Zeolitas, aunque parece ser que los efectos varían según la raza. Evans \& Farrel (1992) y Oliver (1997) reportaron beneficios significativos en el desempeño productivo de la estirpe de ponedoras con el uso de Zeolita, sin encontrar relación directa con la dieta.

Niveles de uso.- Los niveles de inclusión de Zeolita varían del 1 al 10\%. El nivel recomendado para la Zeolita sintética es el $1 \%$ y para las naturales se han reportado niveles tan altos como el $10 \%$. Estas diferencias en la dosis reflejan las principales características físicas y químicas entre las Zeolitas naturales y sintéticas y el contenido de impurezas en las Zeolitas naturales. Khademi (2003) reportó que hasta $7 \%$ de inclusión de Zeolitas naturales mejoró el consumo y el desempeño de las aves, pero que con niveles superiores se presentó un efecto adverso. Las aves sometidas a estrés calórico se beneficiaron con la inclusión de $1.5 \%$ de Zeolitas sintéticas en la dieta (Ingram et al. 1988).

\section{Discusión y Conclusiones}

La falta de evidencia concreta sobre los efectos de la Zeolita sobre los parámetros productivos, como el crecimiento y la producción de huevos, así como los reportes de que la Zeolita podría producir efectos adversos en la avicultura, generan preguntas sobre la pertinencia de su uso en las dietas para aves. Sin embargo existe evidencia sobre los beneficios de la Zeolita en la eficiencia alimenticia, la utilización de nutrientes, la prevención de la aflatoxicosis y la reducción de la humedad y olor en la cama de las aves (Turan 2007, Shariatmadari 2008). Es importante mencionar que la Zeolita tiene un efecto positivo cuando hay una deficiencia de nutrientes, así como en condiciones de temperaturas altas, cuando el alimento puede contener aflatoxinas.

Uno de los mayores problemas encontrados al revisar la literatura sobre la aplicación de Zeolitas fue la falta de detalles completos sobre las características con importancia para los experimentos agrícolas. Tal omisión ha dificultado la interpretación y reproducción de resultados experimentales. Evans (1989) mencionó diez criterios que son deseables para la caracterización de las Zeolitas, los cuales deberían ser reportados en los experimentos agrícolas; estos son: nombre de la especie de Zeolita utilizada, nombre y dirección del proveedor, código del producto, tamaño de partícula, composición mineralógica, composición química, homogeneidad del material, tamaño del cristal, propiedades de intercambio catiónico y una descripción de modificaciones hechas al material.

En conclusión, la variabilidad encontrada en los resultados reportados en la literatura y los posibles efectos adversos debidos al uso de la Zeolita, además de la dificultad para balancear dietas formuladas con dosis mayores de este componente, deben ser tenidos en cuenta por los avicultores a la hora de tomar la decisión de aplicar o no las Zeolitas en la dieta que suministran a sus aves.

\section{Literatura citada}

Bartko, P., H. Seidol \& G. Kovac. 1995. Use of clinoptilolite rich tuffs form Slovakia in animal production: En: Douglas, W.M. \& F.A. Mumpton (Eds). 1995. Occurrence, properties and use of natural Zeolites. Brockport, New York. 467pp.

Edwards, H.M. Jr., M.A. Elliot, \& S. Soocharenying. 1992. Effect of dietary calcium and tibia dyschondroplasia: interaction with light, cholecalciferol, 1,25-dihydroxycholecalciferol, protein and synthetic Zeolite. Poultry Science 71(2): 2041-2055

Elliot, M.A. \& H.M. Edwards. 1991. Some effects of dietary aluminium and silicon in broiler chickens. Poultry Science 70: 1390-1402

Elliot, M.A., B.S. Edwards, \& J. Burdette. 1990. Comparison of the effect of synthetic and natural Zeolite on laying and broiler chickens' performance. Poultry Science 70: 2115-2130

Evans, M. 1989. Zeolites-Do they have a role in 
poultry production?. En: Farrell. D.J. (Ed). 1989. Recent Advances in Animal Nutrition in Australia. p. 245-268.. University of New England. Armidale, Australia

Evans, M. \& D.J. Farrell. 1992. No effect of natural or synthetic Zeolite on performances and egg shell quality of laying hens. Poultry Science 71: 336

Fetheire, R.R, D.Milles \& R.H. Harms. 1988. The influence of dietary phosphorous level in laying hen diets containing ethical feed components. Poultry Science 67:86

Gottardi, G. \& Galli, E. 1985. Natural Zeolites. Springer-Verlag, Berlin, Germany. 409 pp.

Huff, W.E., L F. Kubena, R.B. Harvey,M.H. Elissalde, A.G. Yersin, T.M. Philips \& G.F. Rottinghaus. 1988. Toxic synergism between aflatoxin and T-2 toxin in broiler chickens. Poultry Science 67: 1418-1423

Ingram, D.R., C.E. Kling, \& S.M. Laurent. 1988. Influence of ethical feed component on production parameters with leghorn hens during high ambient temperature. Nutrition-ReportsInternational 37: 811-818

Khademi, S. 2003. The effect of processing, level and type of natural Zeolite on broiler performances. Msc Thesis. Tarbiat Modares University. Irán.

Kithome, M., J.W. Paul \& A.A. Bomke, 1999. Reducing nitrogen losses during stimulated composting of poultry manure using absorbents or chemical amendments. Journal of Environmental Quality 28: 194-201.

Kubena, L.F. \& R.B. Harvey. 1991. Effect of hydrated sodium calcium aluminosilicate on growing turkey poults during aflatoxicosis. Poultry Science 70:1823-1831

Kubena, L.F., R.B. Harvey, W.E. Huff, M.H. Elissalde, A.G. Yersin, T.D. Philips \& G.F. Rottinghaus. 1993a. Efficacy of hydrated sodium calcium aluminosilicate to reduce to the toxicity of aflatoxin and diacetoxyscripenol. Poultry Science 75: 51-62

Leach, R.H., Heinrichs, B.S. and Burdette, J. (1990) Broiler chickens fed low calcium diets. 1) Influence of Zeolite on growth rate and parameters of bone metabolism. Poultry Science 69: 1534-1543

Miles, R.D., R.H. Harms \& S.M. Lourent. 1986. Influence of sodium Zeolite on laying hens performance. Nutrition-Reports International 34: 1097-1103

Mumpton, F.A. \& P.H. Fishman. 1977. The application of natural Zeolites in animal science and aquaculture. Journal of Animal Science 45:
1188-1203

Nakaue, H.S. \& U.K. Koelliker. 1981. Studies with clinoptilolite in poultry. Effect of feeding varying levels of clinoptilolite (Zeolite) to dwarf single comb white leghorn pullets and ammonia production. Poultry Science 60: 944-949

Nestorov, N. 1984. Possible application of natural Zeolite in animal husbandry. En: Pond, W.G. \& F.A. Mumpton (Eds.)1984. Zeo Agriculture: Use of Natural Zeolites in Agriculture and Aquaculture. p. 129. Westview Press Boulder, Colorado, U.S.A.Oliver, M.D. 1989. Effect of feeding clinoptilolite (Zeolite) to three strain of laying hens. British Poultry Science 30: 115-121

Oliver, M.D. 1997. Effect of feeding clinoptilolite (Zeolite) on the performances of three strain of laying hens. British Poultry Science 38: 220223

Petunkin, N. 1991. Influence of Zeolites on animal digestion. En: Occurrence, Properties and Utilization of Natural Zeolites. Fuentes, G.R. \& J.A. González (Eds). 1991. Memorias de la 3ra. Conferencia sobre Ocurrencia, Propiedades y Usos de las Zeolitas Naturales. Abril 9-12. La Habana. Cuba. p: 280. Cuba.

Pond, N.G. \& J.T. Lee. 1984. Physiological effects of clinoptilolite and synthetic Zeolite A in animals. En: Pond, W.G. \& F.A. Mumpton (Eds.)1984. Zeo Agriculture: Use of Natural Zeolites in Agriculture and Aquaculture. p. 129. Westview Press Boulder, Colorado, U.S.A.

Pond, W.G., D.C. Church \& K.R. Pond. 1995. Basic animal nutrition and feeding. John and Sons, Inc., USA.

Ramous, A.G \& E.Gounzales, 1997. Prevention of aflatoxicosis in farm animals by means of hydrated sodium calcium aluminosilicate addition to feedstuffs: a review. Animal Feed Science and Technology 65: 197-206

Roland, D.A. 1988. Further studies of effects of sodium aluminosilicate on egg shell quality. Poultry Science 67:577-584

Roland, Sr. D.A., M. Farmer \& D. Marple. 1985. Calcium and its relationship to excess feed consumption, body weight, egg size, fat deposition, shell quality, and fatty liver hemorrhagic syndrome. Poultry Science 64: 2341-2350

Roussel, J.D., J.K.Thibodeaux, R.W. Adkinson, G.M. Toups \& L.L. Goodeaus. 1992. Effect of feeding various level of sodium Zeolite A on milk yield, milk composition and blood profiles in the thermally stressed Holstein cows. International Journal of Veterinary Nutrition Research 62: 91-97 
Shariatmadari, F. 2008.The application pf Zeolite in poultry production. World's Poultry Science Journal 64:76-84

Turan, G.N. 2007.The application of Zeolite in poultry production. Bioresource Technology 99: 2097-2101
Vest, L. \& J. Shutze. 1984. Influence of feeding Zeolite to poultry under field conditions. En: Pond, W.G. \& F.A., Mumpton (Eds.). 1984. Zeo Agriculture: Use of Natural Zeolites. Agriculture and Aquaculture. p. 211. Westview Press, Boulder, Colorado, U.S.A.

Recibido: 12 de octubre de 2009 Aceptado: 26 de abril de 2010 